\title{
Ornstein-Uhlenbeck Process via Conflated Drive of Brownian Motion and Lévy Process and its Application
}

\author{
Rasaki Olawale Olanrewaju Thierno Souleymane Barry Abdisalam Hassan Muse Alex Habineza \\ Pan African University Institute for Basic Sciences, Technology and Innovation (PAUSTI) \\ PO box 62000-00200, Nairobi, Kenya
}

\begin{abstract}
Non-linear time series and linear models were not designed to detect probabilistic process that are depict by velocity and drift associated to returns the way Ornstein-Uhlenbeck stochastic process describes diffusion and velocity associated to series or waves influenced by Brownian motion or Lévy process. In this research, Brownian motion and Lévy process were conflated as driving force for Ornstein-Uhlenbeck process with its solution applied to Naira-Dollar exchange rates from 2009-2019. The drift and diffusion estimates for the Ornstein-Uhlenbeck process driven by Brownian motion and Lévy process are realization of AR (1) with 2.991 and 0.1672 respectively. The AR(1) realization for the Ornstein-Uhlenbeck process was stationary with estimate $|-0.7204|$ that lies outside the unit circle. The AIC, BIC, RMSE, and MSE for the Ornstein-Uhlenbeck process were estimated to be 483.7572, 483.4782, 0.00101, and 8.395 respectively, compare to estimates of the same indexes for AR (1) of $767.5,634.09,0.3819$, and 23.48. The criterion via the residuals from the Ornstein-Uhlenbeck process was smaller,
\end{abstract}

which connotes that the errors approximated in using drift, Brownian motion and $x_{t-1}$ to estimate $x_{t}$ is relatively small via the Ornstein-Uhlenbeck process.

Keywords: Brownian motion; Drift; Diffusion; Lévy process; Ornstein-Uhlenbeck Process

DOI: $10.7176 / \mathrm{MTM} / 11-3-02$

Publication date:May $31^{\text {st }} 2021$

\section{INTRODUCTION}

Dynamical systems have been a vital modeling in the fields of mechanics, engineering, physics, finance, statistics, physical and social sciences with two realistic traits that are of great interest; the stochastic that consist of the observational outputs that are rackety function of the stimulus such that the forces itself will be galvanized by some latent noise processes; and been qualified by finite-dimensional inner state that are indirect discernible but synopsize at time " $t$ " all entropy about the traits of the procedure applicable to prediction (m-step prediction) (Muzycha \& Vaninsky, 2011; Roweis \& Ghahramani, 2010). Most physical processes in real life involves a flow of event in time and space, especially those exhibiting high variability, such events in real life are referred to as a dynamical system (Olanrewaju, 2018).

Borovkova et al. (2003) asserted that mathematical modeling of economics and financial processes lead, in general, to non-linear deterministic and stochastic dynamical systems in complex structures and surprising behaviors. There is now a well-developed model for characterizing all sorts of attractions, and bifurcations as their parameters are varied. Systems with strong elements or many interacting variables will require further connections to be made between non-linear dynamic proper and statistical mechanics (Kleeman, 2011). There are many examples of dynamics phenomena in nature, which can be regarded as stochastic process. For instance, some of the hydrodynamic phenomena such as rainfall, stochastic dynamical system models are mathematical model and are difficult to identify from data (Ldeo, 1997; Bibbona et al, 2008). However, mathematical model gives stochastic dynamical system model an advantage over non-linear time series model in characterizing the phenomena, with great analytical tools developed in Markov diffusion theory (Olanrewaju, 2018). Markov process is a vital tool for analyzing dependent random Brownian events (Otherwise known as Weiner Process) and Lévy process.

Ornstein-Uhlenbeck (OU) process as described by Ornstein \& Uhlenbeck (1930) is a probabilistic process that depicts the velocity and diffusion (drift) of a Brownian motion or Weiner process influenced by friction. According to Önalan (2009) and Khansari-Zadeh \& Billard (2011), the OU process can be conceived as either a discrete-time or a continuous-time drift of a phenomenon. In other words, is a mathematical model that provides true and accurate representations of a dynamical process of a stationary stochastic process (Donado et al., 2017).

The OU stochastic process is a resonance and noise-induced transition with three random processes; stationary Gauss (Gaussian process), Markov process, and temporal homogeneity of the Brownian or diffusion particles (Kyprianou, 2006). The mathematical representation of the stochastic process can also either be a linear or non-linear dynamical systems. The Ornstein-Uhlenbeck process adopted the non-linearity dynamical systems from the inherited of the non-linearity of the second trait (Markov process) due to its noisy drift, thresholds, transitions and space patterns (Mallick \& Marcq, 2004). The non-linear dynamical systems absolved and inter- 
correlated with the OU process because of the interplay of noisy non-linearity by the Markov random process phenomena in both the theoretical and practical study. The advantage of the non-linear system in the OU process renewed and revitalized the Langevin's description of Brownian motion in fields likes bimolecular study, electronic system, mechanical system (random interaction), financial transitions, and spatial analysis (HernandezMachado et al., 1984). The non-linearity dynamical systems in OU process can be defined via Langevin-stochastic random process by additive or multiplicative noise of either an Ordinary Differential Equation (ODE) or approximation of equations.

The non-linear dynamic based models are stochastic processes that ranges from discrete-time dynamical systems with hidden state, learning stability and variations via Gaussian Mixture models, Kalman Filter; OU and Laguerre stochastic processes; Squared Radial Ornstein-Uhlenbeck stochastic diffusion processes; Gaussian Process Approximations of Stochastic Differential Equations etc. (Bibbona et al, 2008; Uhlenbeck \& Ornstein, 1930). All but two of the aforementioned processes are ODEs based expect for OU and learning stability and variations via Gaussian Mixture models that are processes for low-filtered white-noise models with stochastic error (error term subjected to time series models). There is need for non-linear dynamic OU process driven by Lévy process embedded in Brownian process coupled with a data driven analysis.

The problem of modeling disturbance phenomena via systems of equation has been increasing alarming. Apart from the fact that continuous paths or stochastic observational series of dynamics of linear systems are usually used in place of dynamics of non-linear systems. The stochastic white noises for the processes of driven Ornstein-Uhlenbeck model are conventionally the Brownian (Weiner) and Levy processes. Sometimes, Laguerre stochastic process is usually adopted. No distinct of the aforementioned processes have been pinpointed to be ideal dynamical systems that are non-linear in nature for a better model performance and adequacy. It is in this light; this research work would be employing the Newton-Raphson iterative procedure via the Quasi Maximum likelihood (QML) method of parameter estimation to estimate embedded parameters. The Naira-Euro exchange rate will be time varying series to be used in validating the aforementioned estimates.

\section{METHOD}

Dynamical driven processes (Weiner and Lévy processes) in this study are considered as the forces for arriving at a better OU model ideal performance in terms of parameters, adequacy, as well as better prediction using a continuous path or time experimental data. To capture the dynamical driven processes of the systems, both the driven processes and OU model will adopt the Gaussian probability distribution of stochastic systems. The distribution can be approached from the angle of probability measure. This approach will give a better understanding of the complexity of the behavior of the non-linear dynamical systems. Given an example of system of equations below:

$$
\begin{array}{cc}
\frac{\partial x_{1}}{\partial t} & =g_{1}\left(x_{1}, x_{2}, \cdots, x_{n}, t\right) \\
\frac{\partial x_{1}}{\partial t} & =g_{2}\left(x_{1}, x_{2}, \cdots, x_{n}, t\right) \\
\vdots & \vdots \\
\frac{\partial x_{n}}{\partial t} & =g_{n}\left(x_{1}, x_{2}, \cdots, x_{n}, t\right)
\end{array}
$$

The variables given by $X=\left(x_{1}, x_{2}, \cdots, x_{n}\right)$ at the right hand sides of equation (1) of each derivative function with respect to " $t$ " evolving around with time. All in all, the rate of change depends on the measurement of other variables, if otherwise, that is, in a non-linear way, then it will be referred to as non-linear dynamic system (Strogatz, 1994; Kleeman; 2011).

\subsection{Stochastic Process}

According to Valdivieso et al. (2009), random process $X=(X(t))$ can be referred to as an OU process if it meets the stochastic differential equation below:

$$
X=\left\{\begin{array}{l}
\Delta X(t)=-\delta X(t) \partial t+\sigma \Delta B(t) \\
X(0)=X_{0}
\end{array}\right.
$$

Where $\delta$ and $\sigma$ are stringently positive intensity parameters and $X$ is the independent random variable of the standard Brownian motion of $\mathrm{B}=\{B(t)\}$. 


\subsection{Ornstein-Uhlenbeck (OU) Process Determined by Brownian Motion}

A non-dynamical process via Brownian motion for a single dimensional (that is a AR (1) like Markov-Gaussian could be set as a solution to the stochastic differential equation in (2) as

$$
\Delta X(t)=-\delta X(t) \partial t+\sigma \Delta B(t)
$$

If the rate of interest at time " $\mathrm{t}$ " \& " $\mathrm{k}$ " is a reference point for the rate of interest, then

$$
\Delta X(t)=-\delta(X(t)-k) \partial t+\sigma \Delta B(t) ; \quad X_{0}=x_{o}
$$

\subsection{Ornstein-Uhlenbeck (OU) Process Determined by Lévy Process}

From equation (2)

$$
X=\left\{\begin{array}{l}
\Delta X(t)=-\delta X(t) \partial t+\sigma \Delta B(t) \\
X(0)=X_{0}
\end{array}\right.
$$

Integrating with respect to " $t$ "

$$
\begin{aligned}
& \int_{0}^{t}(\Delta X(t)=-\delta X(t) \partial t+\sigma \Delta B(t)) \\
& X(t)=e(-\delta t)\left(X_{0}+\sigma \int_{0}^{t} e(\delta t) \Delta B(s)\right)
\end{aligned}
$$

For " $s "$ of the standard Brownian motion that is a Markov-Gaussian process with auto-covariance function $V(s, t)=E\left(X_{s}, X_{t}\right)$.

\section{PARAMETER ESTIMATION OF THE CONFLATED DRIVEN PROCESSES OF BROWNIAN AND} LÉVY

If a Lévy process is such that $X\left(t_{0}\right), X\left(t_{1}\right), X\left(t_{2}\right), \cdots, X\left(t_{n}\right)$ are sample of the stationary process $x_{0}, x_{1}, x_{2}, \cdots, x_{n}$ such that their observed values is $y_{t}=\theta_{1} x_{t-1}$. Then the transition density of the OU process at one-dimensional (that is, at autoregressive of order one) via Markovian and Gaussian property is as:

$g\left(x, t / y, s, \sigma^{2}\right)=\frac{e-\left(\frac{\left(x_{t}-y e^{-\frac{(t-s)}{\delta}}\right)^{2}}{\sqrt{\sigma^{2} \delta\left(1-e^{-\frac{2(t-s)}{\delta}}\right)}}\right)}{\sqrt{\pi^{2} \sigma^{2} \delta\left(1-e^{-\frac{2(t-s)}{\delta}}\right)}} \ni x_{t} \in(-\infty, \infty)$

$$
g\left(x, t / \theta_{1}, x_{t-1}, s, \sigma^{2}\right)=\frac{e-\left(\frac{\left(x_{t}-\theta_{1} x_{t-1} e^{-\frac{(t-s)}{\delta}}\right)^{2}}{\pi \sqrt{\sigma^{2} \delta\left(1-e^{-\frac{2(t-s)}{\delta}}\right)}}\right)}{\sqrt{\sigma^{2} \delta\left(1-e^{-\frac{2(t-s)}{\delta}}\right)}}
$$


Shirking the parameters in the likelihood function into a parameter space

$$
\Theta=\left\{\theta_{1}, \sigma^{2}, \delta\right\}
$$

$$
L(\Theta)=\frac{1}{\sqrt{\pi \sigma^{2}}} e-\left(\frac{x_{0}^{2}}{\sigma^{2}}\right) \prod_{i=1}^{n} \frac{\left(\frac{\left(x_{t}-\theta_{1} x_{t-1} e^{-\frac{(t-s)}{\delta}}\right)^{2}}{\sqrt{\sigma^{2} \delta\left(1-e^{-\frac{2(t-s)}{\delta}}\right)}}\right)}{\pi \sqrt{\sigma^{2} \delta\left(1-e^{-\frac{2(t-s)}{\delta}}\right)}}
$$

Minimizing the observed the maximum likelihood via log-likelihood function

$$
l(\Theta)=\log L(\Theta)=-\frac{(n+1)}{2} \log \left(\pi \sigma^{2}\right)-\frac{x_{0}^{2}}{2}-\frac{1}{2} \sum_{i=1}^{n}\left(\delta\left(1-\exp \left(-\frac{2(t-s)}{\delta}\right)\right)\right)
$$

The embedded parameters equation (11) will be estimated by adopting the Newton-Raphson iterative procedure for a no close form solution initially.

\section{RESULTS AND DATA ANALYSIS}

\subsection{Empirical Results}

The dataset used to validate the OU process driven by the blended process of Lévy and Brownian motion is the monthly exchange rates of Naira-Dollar from 2009 to 2019. The dataset was extracted from the website of Central Bank of Nigeria. The rates are the ones termed as the standard rates for international currency.

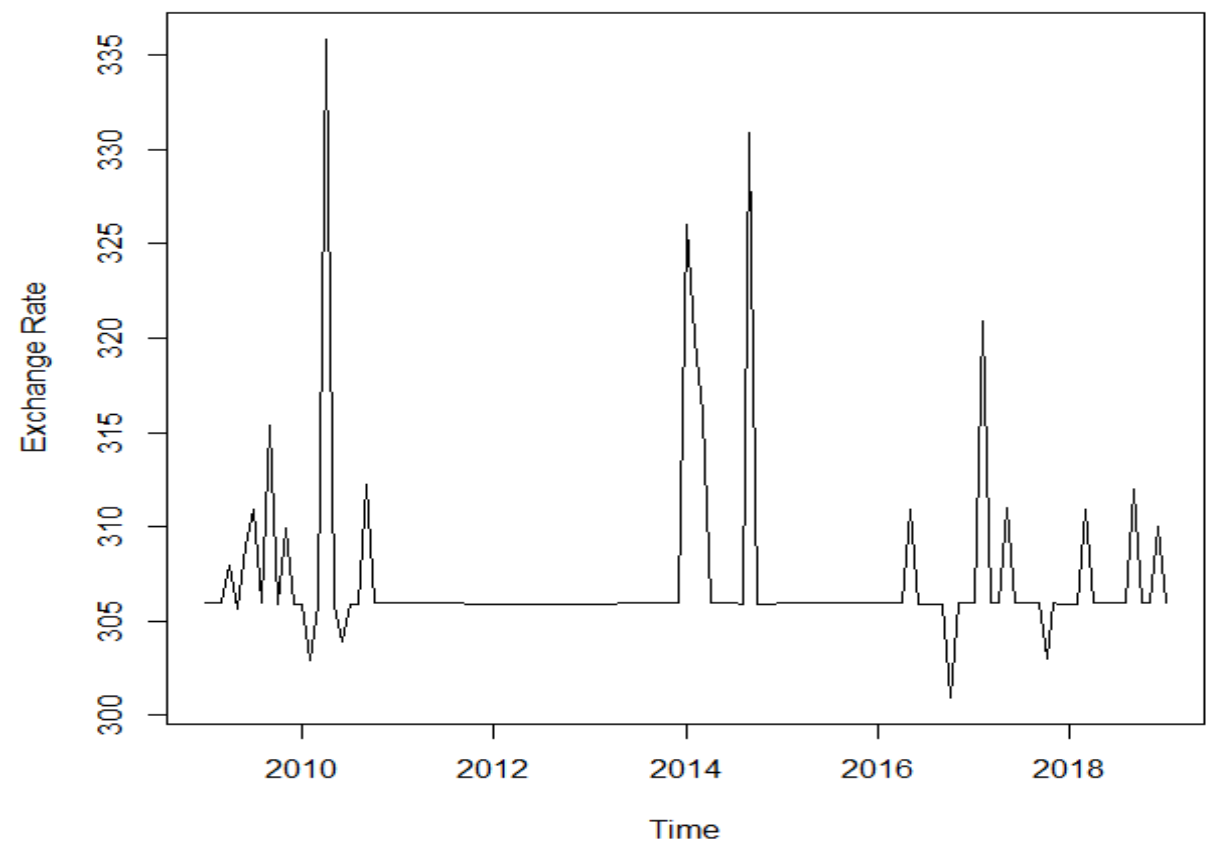

Figure 1: The Time plot of the Ten years Exchange Rate of Naira-Dollar

From the time plot in figure 1 above, it is obvious that the rate started with a moderate exchange between 305 and 315 in the early stage of 2010, then skyrocketed to 336 towards ending of 2010. A continuous slumping in exchange rate was experienced between early 2011 to the beginning of 2014, a spurious jump was experienced at the first quarter of 2014. However, a continuous constant in the exchange rate commenced from the of second quarter of 2014 till second quarter of 2016, before a deteriorating exchange rates were recorded towards ending of 2016. A notable oscillation (zig-zag trend) with upper limit of 310.00 and lower limit of 303 was experienced from the ending of 2017 till 2019. 
Table 1: Descriptive Statistics for the Monthly Exchange Rate of Naira -Dollar

\begin{tabular}{|l|l|l|l|l|l|l|l|}
\hline Indexes & Min. & 1st Qu. & Median & Mean & 3rd Qu. & Max. & S.D. \\
\hline Estimates & 303.8 & 309.9 & 316.0 & 318.6 & 316.89 & 336.0 & 0.004 \\
\hline
\end{tabular}

From table 1 above, the minimum rate over the ten years is 303 and the maximum is 336.0 over the stipulated studied of interest. However, the range is 32.2, while the rates for the ten years were clustered around 318.6. The variations among the exchange rates were estimated to be $0.004(0.4 \%)$ which suggested a less dispersed among the observations. The value of the median conjoined with the value of the mean. The first and third quarters of the rates are 309.9 and 316.89 respectively.

Table 2: Stationary Test and Skewed Coefficient

\begin{tabular}{|l|l|}
\hline Tests/ Coefficients & P-value \\
\hline Box-Pierce test & $<2.2 \mathrm{e}-16$ \\
\hline Skewness & -0.2614616 \\
\hline Kurtosis & -0.6986375 \\
\hline Phillips-Perron (PP) Unit Root Test & 0.02706 \\
\hline Augmented Dickey-Fuller (ADF)Test & 0.01 \\
\hline KPSS Test for Level Stationarity & 0.02902 \\
\hline
\end{tabular}

From table 2, the Kwiatkowski-Phillips-Schmidt-Shin (KPSS) is one of the fundamental tests for trend stationary (stationary around a deterministic trend) of the observable series. The trend is expressed as the sum of deterministic trend, random walk and stationary error, however, since the P-value $=0.02902$ for the KPSS test is less than the $5 \%$ level of the significant level, the observable series of the exchange rate is stationary. The ADF test, which serves as complement test for KPSS yielded a P-value $=0.01$ that is less than $\%$ level of the significant level, with conjoined decision. The Phillips-Perron (PP) unit root test with $\mathrm{P}$-value $=0.02706<0.05$ suggested an integrated lag of greater than one.

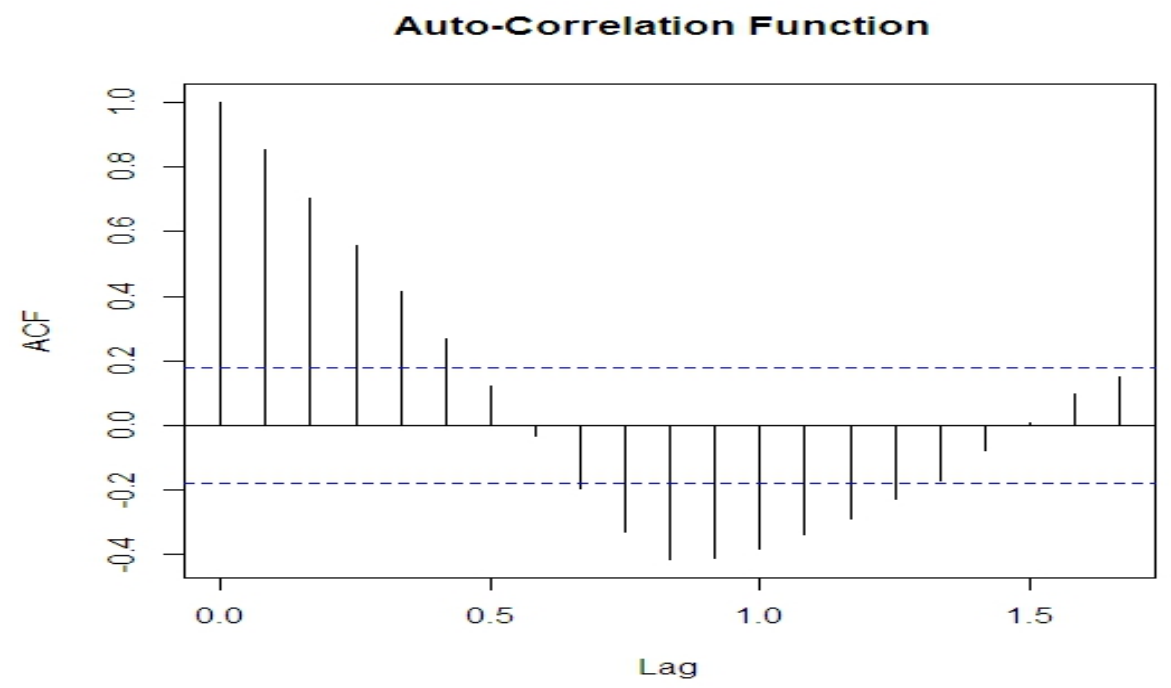

Figure 2: The Auto-Correlation Function 


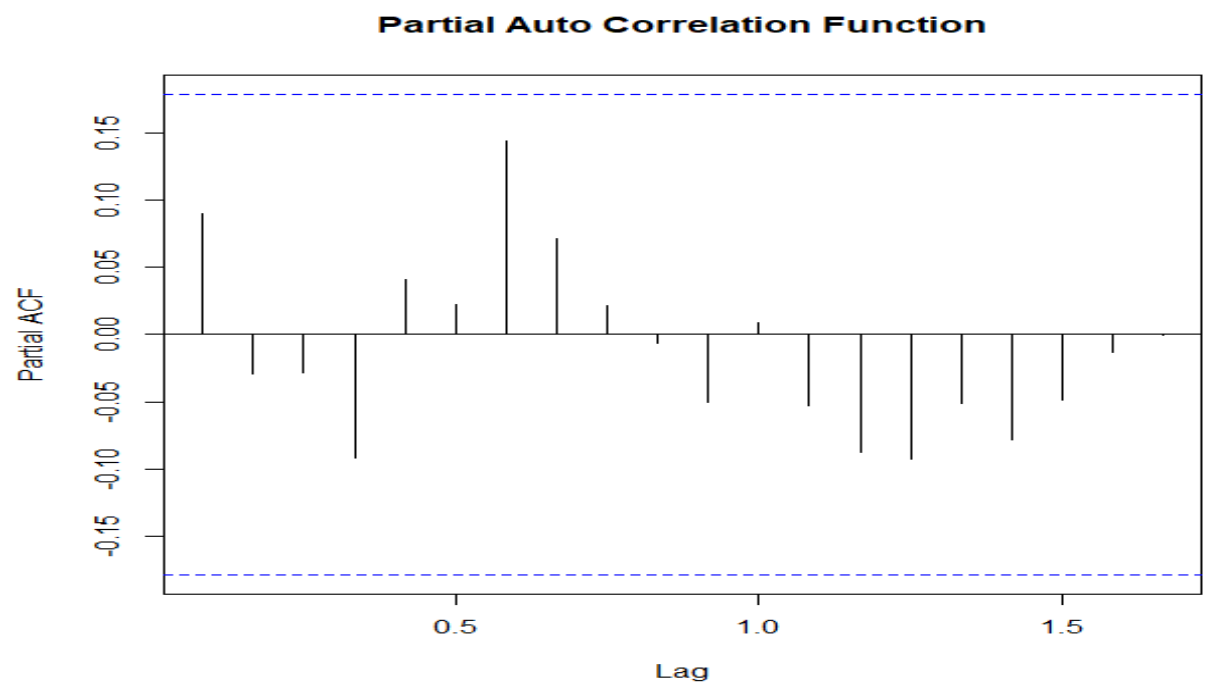

Figure 3: The Partial Auto-Correlation Function

The selection criteria of tentative time series models are normal achieved by matching estimated autocorrelations with the theoretical autocorrelation. The Box and Jenkins ARIMA models can be shown to be optimal and provides a systematic approach to model selection, utilizing all the information contained in the sample autocorrelations (ACF) and partial autocorrelation (PACF) functions. The ACF and PACF are meaningful only when applied to stationary series. The matching of the first twenty (20) estimated sample autocorrelations and partial autocorrelations of the underlying stochastic processes from the Log exchange rate series suggested that the series were stationary, with the ACF and PACF of the resulting series as shown in table 3 belows. The correlelogram for the ACF and PACF of the underlying stochastic processes is as shown in figure 2 and figure 3.

Table 3: Sample ACF, PACF, and AIC for the realization of the Exchange Rates

\begin{tabular}{|l|l|l|l|}
\hline Lag/Order & ACF & PACF & AIC \\
\hline 1 & 0.9787 & 0.9723 & 20.5642 \\
\hline 2 & 0.3724 & -0.0356 & 18.4563 \\
\hline 3 & 0.9546 & 0.9552 & 15.2988 \\
\hline 4 & 0.4560 & 0.0068 & 25.8840 \\
\hline 5 & 0.0345 & -0.0524 & 27.4902 \\
\hline 6 & 0.1890 & 0.0008 & 21.8948 \\
\hline 7 & 0.7056 & 0.3102 & 19.6350 \\
\hline 8 & 0.5039 & -0.066 & 23.6893 \\
\hline 9 & 0.6590 & 0.0034 & 17.088 \\
\hline 10 & 0.5824 & 0.0100 & 18.0452 \\
\hline 11 & 0.3210 & -0.0721 & 28.9086 \\
\hline
\end{tabular}

From the sampled ACF and PACF above, it could be deduced that the lag that supposed to decay exponentially, but usually difficult to assess the order of an AR process from the sampled ACF alone. For higher order process, the ACF maybe a mixture of damped exponential function. However, using the Auto-Correlation Function (ACF), Partial Auto-Correlation Function (PACF), and Alkaike Information Criteria (AIC), it was deduced that the values of the ACF and PACF at lag three (1) has the highest values with 0.9787 and 0.9723 respectively. In addition, the AIC at lag one (1) has the least AIC from the pool of AICs from lag one to lag eleven.

Table 4: The AIC, BIC, RMSE, and MSE for Autoregressive (AR) realization.

\begin{tabular}{|l|l|l|l|l|}
\hline Model & AIC & BIC & RMSE & MSE (\%) \\
\hline AR(1) & 941.45 & 945.89 & 0.0211 & 15.4 \\
\hline AR(2) & 985.7 & 977.1 & 0.0215 & 16.6 \\
\hline AR(3) & 959.8 & 963.56 & 0.0036 & 10.57 \\
\hline AR(4) & 984.3 & 970 & 0.02094 & 17.45 \\
\hline AR(5) & 983.4 & 966.2 & 0.0208 & 13.98 \\
\hline AR(6) & 982.8 & 962.7 & 0.0207 & 19.28 \\
\hline AR(7) & 986.4 & 963.5 & 0.01979 & 16.6 \\
\hline AR(8). & 962.1 & 964.4 & 0.01979 & 14.20 \\
\hline AR(9) & 959.9 & 960.1 & 0.01975 & 14.100 \\
\hline
\end{tabular}


In collaboration with the ideal realization and representation of lag three of the ACF and PACF. The AIC, BIC, RMSE, and MSE yielded the fitted model of AR (1) with all the roots lying outside the unit circle, that is the coefficients $\left|\phi_{1}\right|<1$ (-0.7204) less than 1 .

Table 5: The OU process driven by Brownian motion and Lévy Process Statistics for $X(t)$ at time $t=10$ for the

\begin{tabular}{|c|c|c|c|c|}
\hline \multicolumn{5}{|c|}{ AR (1). } \\
\hline Estimates & Coefficients & & & \\
\hline Drift & 2.9921 & \multicolumn{3}{|c|}{ Log-Likelihood $=475.7572$} \\
\hline Diffusion & 0.1672 & \multicolumn{3}{|c|}{$\mathrm{AIC}=483.7572$} \\
\hline Median & 2.9903 & \multicolumn{3}{|c|}{$\mathrm{BIC}=483.4782$} \\
\hline Mode & 2.9392 & \multicolumn{3}{|c|}{ RMSE $=0.00101$} \\
\hline First quartile & 2.6575 & \multicolumn{3}{|l|}{$\mathrm{MSE}=8.395$} \\
\hline Third quartile & 3.3364 & & & \\
\hline Minimum & 0.9042 & coefficients & Estimate & Std. Error \\
\hline Maximum & 4.5287 & $\phi$ & 0.6387 & 1.1875 \\
\hline Skewness & -0.0220 & $\phi_{2}$ & -0.3606 & 0.1889 \\
\hline Kurtosis & 3.0049 & $\phi_{3}$ & 0.8650 & 0.0354 \\
\hline $3^{\text {th }}$-order moment & 0.0027 & & & \\
\hline $4^{\text {th }}$-order moment & 0.1884 & & & \\
\hline $5^{\text {th }}$-order moment & 0.0144 & & & \\
\hline $6^{\text {th }}$-order moment & 0.2335 & & & \\
\hline C.I $\left(\phi_{1}\right)$ & $(0.3045,0.9241)$ & & & \\
\hline C.I $\left(\phi_{2}\right)$ & $(-0.6248,0.1198)$ & & & \\
\hline C.I $\left(\phi_{3}\right)$ & $(0.0807,0.179)$ & & & \\
\hline
\end{tabular}

$$
X=\left\{\begin{array}{l}
\Delta X(t)=-2.9921 X(t) \Delta t+0.1672 \Delta B(t)-0.7204 X_{t-1} \\
X(10)=X_{0},
\end{array}\right.
$$

The drift and diffusion estimates for the OU driven by Brownian motion and Lévy process of AR(3) realization are;

$$
\Delta t=2.9921, \Delta B(t)=0.1672 \text { respectively. The }\left|\phi_{1}\right|<1 \text { for the AR(1) realization for the OU process are }
$$
stationary also with $|-0.7204|$ lies outside the unit circle. The AIC, BIC, RMSE, and MSE for the OU process are estimated to be $483.7572,483.4782,0.00101$, and 8.395 respectively, compare to estimates of the same indexes for AR (1) of 767.5, 634.09, 0.3819, and 23.48. The criterion via the residuals from the OU process is smaller, which connotes that the errors approximated in using drift, Brownian motion and $x_{t-1}$ to estimate $x_{t}$ is relatively small via the OU process. 
Two Transition Density for the Exchange Rate $\mathbf{t}=1$
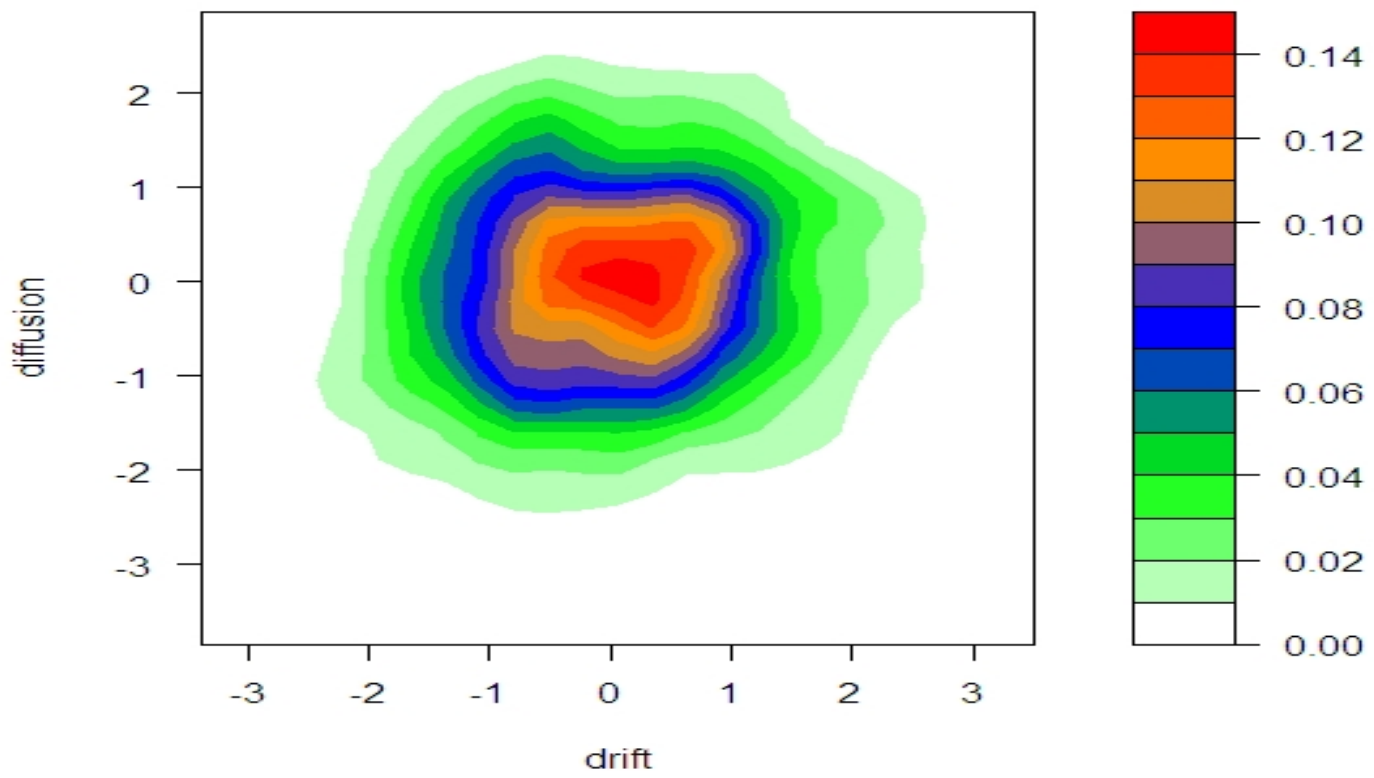

Figure 4: The Transition Density for the OU Process.

From figure 4, the OU process recoded a two switching regimes. It started with the initial state from 0.00 diffusion and started fading at 0.04 and finally faded at 0.06 before the first regime manifested at 0.061 an elapsed 0.08 . The second and the last regime was embarked at 0.10 and finished at 0.14 .

\section{CONCLUSION}

The non-linear time series process via the OU process of OU process embedded by AR (1) with two regimes switching. It started with the initial state from 0.00 diffusion and started fading at 0.04 and finally faded at 0.06 before the first regime manifested at 0.061 an elapsed 0.08 . The second and the last regime was embarked at 0.10 and finished at 0.14 . The criterion via the residuals from the OU process is smaller, which connotes that the errors

approximated in using drift, Brownian motion and $x_{t-1}$ to estimate $x_{t}$ is relatively small via the OU process. Justifiably, the OU process, apart from the fact that it was able to reduce the long memory and noisy part of the Naira-Dollar exchange rates, it catered, unveiled, cognizance and accommodated the five regimes-switching Naira-Dollar exchange rates. The residual of the fitted OU process seemed linear, which is a suggestion of ideal realization without of the chosen fitted model. For it to be ideal, it has satisfied the constant variance assumption and the normality assumption.

Having considered the OU process, it was noted that OU process of AR (3) realization model performed better for the financial data with smallest Minimum AIC, BIC, RMSE, MSE with 483.7572, 483.4782, 0.00101, and 8.395 respectively, compare to estimates of the same indexes for AR(1) of 767.5, 634.09, 0.3819, and 23.48 and ability to capture drift and diffusion coefficients in the unstable financial data. Therefore, OU process, given our findings above, is a more adequate and fitting model to a fluctuating financial time series data.

\section{Recommended Further Researchable Area}

In recommendation of this research work, swinging time varying financial data should not always be subjected to non-cluster varying models like GARCH, APARCH, GJR-GARCH, SETAR etc. because those models won't be able to capture to drift and diffusion.

Further research to be dealt are in the following areas: Moving Average realization of OU process; Subsets of Auto-Regressive realization of OU process; Generalized Auto-Regressive Conditional Heteroscedasticity realization of OU process; Violating the white noise assumption of normality which might lead to either using Generalized Error Distribution (GED) or student-t- distribution for the realization of OU process as an alternative for Gaussian distribution.

\section{Acknowledgement}

Our appreciation goes to the Central Bank of Nigeria for its support in providing the requested dataset used for this research. 


\section{References}

Bibbona, E., Panfilo, G., \& Tavella, P. (2008). The Ornstein-Uhlenbeck process as a model for filtered white noise. Dell'Università di Torino, 45, 117-126. doi:10.1088/0026-1394/45/6/S17.

Borovkova, S., Permana, F., \& Weide, H. V. (2012). American basket and spread option pricing by a simple binomial tree. Journal of Derivatives, 19(4), 29-38.

Donado, G., Leonenko, N. N., Sikorskii, A., \& Taqqu, M.S. (2017). The unusual properties of aggregated superpositions of Ornstein-Uhlenbeck type Processes. Bernoulli, 1-34.

Hernandez-Machado, A., Miguel, M., Sancho, J.M. (1984). Relaxation Time of Processes Driven by Multiplicative Noise. Physics Review A, 29, 33-88.

Khansari-Zadeh, S. M., Billard, A., (2011). Learning stable non-linear dynamical systems with Gaussian mixture models. IEEE Transaction on Robotics, 3 (3), 12-30.

Kleeman, R. (2011). Information theory and dynamical system predictability. Entropy, 13, $612-649$. doi:10.3390/e13030612.

Kyprianou, A. (2006). Introductory lectures on fluctuations of Lévy processes with applications. Springer, 2006.

Ldeo, M.S. (1997). An introduction to dynamical systems and chaos.

Mallick, K., \& Marcq, P., (2004). On the Stochastic pendulum with Ornstein-Uhlenbeck noise. Journal of Physics A: Mathematical and General, 37(17), 47-69.

Muzychka, S.A., \& Vaninsky, K.L., (2011). A class of nonlinear random walks related to the Ornstein-Uhlenbeck process. Markov Processes and Related Fields, 17(2), 277-304.

Önalan, Ö. (2009). Financial Modelling with Ornstein-Uhlenbeck processes driven by Lévy process. Proceedings of the World Congress on Engineering, Vol. II, WCE 2009, London, UK.

Olanrewaju, R.O. (2018). On the Efficiency and Robustness of Commingle Wiener and Levy Driven Processes for Vasciek Model. Mathematical and Computational Sciences, 12(11). waset.org/Publication/10009776.

Roweis, S., \& Ghahramani, Z. (2010). An EM algorithm for identification of non-linear dynamical systems. Gatsby Computational Neuroscience Unit, University College London, London WCIN 3AR, UK, 2010. http://gatsby.ucl.ac.uk.

Strogatz, S.H. (1994). Nonlinear dynamics and chaos: with applications to physics, biology, chemistry, and engineering, Addison-Wesley Publishing Co., Reading, MA.

Uhlenbeck, G.E. \& Ornstein, L.S. (1930). On the theory of Brownian Motion. Physical Review, 36, 823-84.

Valdivieso, L., Schoutens, W., \& Tuerlinckx, F. (2009). Maximum likelihood estimation in processes of OrnsteinUhlenbeck type. Stat. Infer. Stoch Process, Vol.12, 1-19. doi: 10.1007/s11203-008-9021-8. 